\section{Short-term ocular tolerability of dorzolamide $2 \%$ and brinzolamide $1 \%$ vs placebo in primary open-angle glaucoma and ocular hypertension subjects}

WC Stewart ${ }^{1,2}$, DG Day³, JA Stewart ${ }^{1}$, KT Holmes ${ }^{1}$ and JN Jenkins ${ }^{1}$

\begin{abstract}
Purpose To compare ocular tolerability of dorzolamide $2 \%$, brinzolamide $1 \%$, and placebo given three times daily.

Methods A prospective, double-masked, three-centre, crossover comparison in which 25 ocular hypertensive or primary-open angle glaucoma subjects were randomized to receive dorzolamide, brinzolamide, or placebo three times daily for 3 days. Intraocular pressure, visual acuity, a visual analogue scale, and ocular and systemic symptom queries were completed at the end of each period.

Results After chronic dosing, there was a significant difference in ocular pain on the visual analogue scale among the groups at the 10-s postinstillation time point with dorzolamide having the highest level $(22.5 \pm 28.9)$ compared to brinzolamide $(5.0 \pm 8.7)$ or placebo $(3.2 \pm 10.4)(P=0.0006)$.

No differences between groups were observed preinstillation nor following dosing at 3 or 10-min postinstillation. On the initial instillation, the 10-s postinstillation pain was rated as $43.3 \pm 77.1$, which was significantly higher than after chronic dosing $(P=0.017)$. On the ocular symptom query, dorzolamide had the highest incidence of burning/stinging and redness compared to the other groups, but was generally characterized as mild. There were no significant differences in the visual acuity at any time point.

Conclusions This study suggests that subjects treated with dorzolamide suffer more ocular pain upon instillation compared to
\end{abstract}

brinzolamide or placebo. However, pain symptoms are fewer following chronic dosing and are generally characterized as mild. Eye (2004) 18, 905-910. doi:10.1038/sj.eye.6701353 Published online 27 February 2004

Keywords: ocular tolerability; dorzolamide; brinzolamide

\section{Introduction}

Dorzolamide $2 \%$ is a topical carbonic anhydrase inhibitor that was commercially released several years ago by Merck \& Co., Inc. to provide an alternative to the oral carbonic anhydrase inhibitor, acetazolamide, in the treatment of elevated intraocular pressure. Dorzolamide has been a popular adjunctive agent and is used not infrequently as monotherapy. Dorzolamide reduces the intraocular pressure by $10-26 \%$ as a monotherapy agent and has eliminated most of the systemic symptoms commonly associated with acetazolamide. ${ }^{1-5}$ To date, bone marrow depression has not been reported and kidney stone incidence has not been elevated above the expected level for the general population with dorzolamide use. ${ }^{4,5}$ Several side effects do occur commonly with dorzolamide, however, including bitter taste, stinging, ocular pain on instillation, and conjunctival hyperemia. These side effects generally are not severe enough to discontinue use of the medicine. ${ }^{4,6}$

Brinzolamide $1 \%$ is manufactured by Alcon and is a topical carbonic anhydrase inhibitor
${ }^{1}$ Pharmaceutical Research Network

LLC, Charleston, SC, USA

${ }^{2}$ Carolina Eye Institute at the University of South Carolina School of Medicine Columbia, SC, USA

${ }^{3}$ Atlanta Research Company Atlanta, GA, USA

Correspondence: WC Stewart Pharmaceutical Research Network, LLC 1639 Tatum Street Charleston, SC 29412-2464, USA Tel: + 8437626500 Fax: + 8437627444

E-mail: prnc@

bellsouth.net

Received: 1 July 2003 Accepted: 21 October 2003 Published online: 27 February 2004

This study was sponsored by an unrestricted grant from Merck, Inc. None of the authors have a proprietary interest in Merck or in any of the commercial products mentioned in the paper. 
that has been commercially released more recently. Studies have shown that it reduces the intraocular pressure as a monotherapy agent by $15-21 \%$ and as an adjunctive agent added to timolol maleate by $13-16 \%{ }^{7,8}$ It can be given b.i.d. or t.i.d. but when used t.i.d. less acute pain on instillation with brinzolamide $v s$ dorzolamide from subject reporting has been noted. ${ }^{9}$ Insufficient information still exists, however, which quantitates or characterizes the comfort of these two topical agents.

The purpose of this trial was to compare short-term tolerability of dorzolamide $2 \%$ and brinzolamide $1 \%$ vs placebo in primary open-angle and ocular hypertensive subjects using a numeric-based visual analogue pain scale and solicited adverse events by subject survey.

\section{Materials and methods}

\section{Subjects}

Subjects must have fulfilled the following conditions to qualify for enrollment into the trial: 18 years of age or older, willing to comply with the investigator's and protocol's instructions, subject's signature on the informed consent document, have a clinical diagnosis of primary open-angle, pigment dispersion, or exfoliation glaucoma, or ocular hypertension in at least one eye (study eye). At screening the intraocular pressure must have been considered to be controllable on one drug therapy in the study eye(s) in such a way that should assure clinical stability of vision and the optic nerve throughout the trial. In eyes not included in the study, the intraocular pressure should have been able to be controlled on no pharmacologic therapy or on the study medicine. Visual acuity must have been 20/200 or better in the study eye(s).

Subjects with any of the following conditions on the eligibility exam were not enrolled into the trial: any abnormality preventing reliable applanation tonometry in study eye(s), contact lens use during the trial in the study eye(s), any opacity or subject uncooperativeness that restricted adequate examination of the ocular fundus or anterior chamber in the study eye, any concurrent infectious/noninfectious conjunctivitis, keratitis or uveitis in either eye, any history of allergic hypersensitivity or poor tolerance to any components of the preparations used in this trial including sulpha medications, female subjects of child-bearing potential not using reliable means of birth control, pregnant or lactating females, any clinically significant, serious, or severe medical or psychiatric condition, participation (or current participation) in any investigational drug or device trial within the previous 30 days prior to the baseline (Visit 2) of this trial, intraocular conventional surgery or laser surgery within the past 3 months in the study eye(s), according to the investigator's best judgment risk of visual field or visual acuity worsening as a consequence of participation in the trial, inability to understand the trial procedures, and thus inability to give informed consent, progressive retinal or optic nerve disease apart from glaucoma, past history of bone marrow depression or aplastic anaemia, or advanced corneal endothelial cell loss.

\section{Procedures and evaluation}

At the screening visit (Visit 1), each subject signed an Institutional Review Board approved informed consent. Afterwards, subjects had an ocular and medical history obtained and inclusion/exclusion criteria were reviewed. Subjects then had an ocular examination performed, as well as at each subsequent visit, which consisted of slitlamp biomicroscopy, ETDRS visual acuity, and Goldmann applanation tonometry. At the screening visit subjects also had dilated funduscopy and visual field testing performed.

After the screening visit, subjects were scheduled to return for Visit 2 and washed out of their current medication for 3 days. Since the primary efficacy variable for this study was not intraocular pressure, the washout period was approximately 3 days to reverse any extraocular or systemic effect from the previous medication.

At Visit 2, baseline subjects were randomized to receive one of three study medicines: dorzolamide $2 \%$ (Trusopt $^{\circledR}$, Merck \& Co., Inc., Blue Bell, PA, USA), brinzolamide 1\% (Azopt ${ }^{\circledR}$, Alcon, Fort Worth, TX, USA), or placebo (Hypotears ${ }^{\circledR}$, CIBA Vision Ophthalmics, Atlanta, GA, USA) in a randomized fashion. The dosing coordinator instilled an initial drop of study medicine in association with the visual analogue scale.

The visual analogue scale was performed by asking subjects to indicate on an unmarked $100 \mathrm{~mm}$ line the intensity of their pain before instillation then at $10 \mathrm{~s}$, $3 \mathrm{~min}$ and $10-\mathrm{min}$ after instillation. A mark of ' 0 ' represented no sensation while ' 100 ' indicated the worst imaginable pain. The location of the mark on the line then was measured with a millimetre rule to provide a numeric score. ${ }^{10}$ Ocular symptom visual analogue scales then were performed also to characterize any discomfort including: ocular dryness, tearing, burning, crusting, itching, gritty sensation, deep pain, and irritation. The visual analogue scale is a validated pain scale that has been described previously for ocular use. ${ }^{10,11}$

Following the visual analogue scale, each subject was instructed to instill one drop of the study medicine at 0800,1600 and at bedtime into the study eye(s). The study was double masked. Subjects were told not to dose 
the morning prior to the next visit and to return to clinic in 3 days.

At the end of the first period (Visit 3), subjects were evaluated for adverse events and compliance, and an ocular and systemic symptom assessment were performed. The unmasked dosing coordinator instilled the study medicine and visual acuity and visual analogue scale were measured. The visual acuity was measured at 1, 5 and $12 \mathrm{~min}$ after instillation and every $30 \mathrm{~min}$ if required until within one line of baseline. The intraocular pressure was then measured. The masked study medicine was collected and the subject scheduled to return in 4 days for the second period.

At the beginning of Period 2 (Visit 4), baseline procedures were again performed as at Period 1 (Visit 2). Patients then dosed their Period 2 masked medication for 3 days and returned for the end of Period 2 assessments (Visit 5). Patients were again washed out of their medication for 4 days and had a baseline evaluation for Period 3 (Visit 6). Patients then dosed their Period 3 masked medication for 3 days and returned for the end of the Period 3 assessments (Visit 7). Subjects received their masked study medicine in an order determined by a Latin square randomization technique. Following the third period, subjects were discontinued from the trial barring any unresolved adverse events.

\section{Statistical analysis}

Pharmaceutical Research Network, LLC, analysed the data. All data analyses were two-sided and a 0.05 alpha level was used. The primary safety variable was visual analogue scale. This was analysed by a one-way analysis of variance for intragroup analysis. ${ }^{12} \mathrm{~A}$ standard deviation of 20 was assumed. This study provided an $80 \%$ power that a difference of 10 could be excluded between groups. ${ }^{11}$ The study was powered using a paired $t$-test model.

Secondary safety parameters for intragroup analysis were evaluated with a $\chi^{2}$ test including adverse events and the ocular as well as systemic symptom queries. ${ }^{13}$ Intraocular pressure and visual acuity (ETDRS) were analysed by a one-way analysis of variance. ${ }^{14}$

\section{Results}

\section{Subjects}

We included 25 subjects in this study with an average age of $57.3 \pm 11.4$ years. Nine subjects were African American and 16 were Caucasian. In total, 11 subjects were male and 14 were female. Four subjects had glaucoma and 21 had ocular hypertension.

\section{Visual analogue scale}

The results of the visual analogue scale are shown in Tables 1 and 2. Figures 1 and 2 show a significant difference in pain scores between dorzolamide, brinzolamide, and placebo at $10 \mathrm{~s}$ after instillation for both acute and chronic dosing. There was no significant difference in mean pain levels among the three groups at the baseline and the

3-min as well as the 10 -min postinstillation time points. Dorzolamide demonstrated the highest level of pain at the 10 -s postinstillation time point $(P=0.0006)$.

Additionally, the change from baseline for dorzolamide compared to brinzolamide and placebo was statistically different at the 10 -s postinstillation time point $(P=0.003)$.

The visual analogue scale pain scores from the initial drop instilled on the first day dosing are also shown in Tables 1 and 2. Similar to after chronic dosing $10 \mathrm{~s}$ after dosing was the only single time point when dorzolamide was higher than brinzolamide or placebo in the mean pain scores or changed from baseline $(P=0.004)$. The pain on the initial instillation was higher than after 3 days of chronic dosing $(P=0.017)$.

\section{Symptom surveys}

Results of the ocular symptom query are shown in Table 3. There was a significant difference for burning/

Table 1 Mean levels from the visual analog scale (mm)

\begin{tabular}{lrlll}
\hline & Dorzolamide & Brinzolamide & Placebo & P-value \\
\hline Chronic (after 3 days of dosing) & & & \\
Baseline & $7.9 \pm 14.1$ & $3.5 \pm 6.6$ & $3.1 \pm 9.5$ & 0.21 \\
$10 \mathrm{sec}$ & $22.5 \pm 28.9$ & $5.0 \pm 8.7$ & $3.2 \pm 10.4$ & 0.0006 \\
$3 \mathrm{~min}$ & $8.2 \pm 12.1$ & $3.7 \pm 7.5$ & $3.0 \pm 10.0$ & 0.15 \\
$10 \mathrm{~min}$ & $6.5 \pm 11.4$ & $3.1 \pm 9.7$ & $2.9 \pm 10.0$ & 0.38 \\
& & & & \\
Acute (initial dose) & & & & \\
Baseline & $5.0 \pm 12.2$ & $2.9 \pm 9.9$ & $4.7 \pm 10.2$ & 0.75 \\
$10 \mathrm{sec}$ & $43.3 \pm 77.1$ & $5.9 \pm 12.8$ & $4.2 \pm 10.0$ & 0.004 \\
$3 \mathrm{~min}$ & $6.1 \pm 10.7$ & $3.9 \pm 10.2$ & $3.9 \pm 9.5$ & 0.70 \\
$10 \mathrm{~min}$ & $5.3 \pm 11.1$ & $3.0 \pm 8.2$ & $4.1 \pm 9.5$ & 0.70 \\
\hline
\end{tabular}

Table 2 Change from baseline on visual analog scale (mm)

\begin{tabular}{lrrrl}
\hline & Dorzolamide & Brinzolamide & Placebo & P-value \\
\hline Chronic (after 3 days of dosing) & & & \\
$10 \mathrm{~s}$ & $14.6 \pm 27.2$ & $1.6 \pm 3.9$ & $0.1 \pm 1.6$ & 0.003 \\
$3 \mathrm{~min}$ & $0.3 \pm 10.2$ & $0.3 \pm 3.4$ & $-0.0 \pm 1.4$ & 0.98 \\
$10 \mathrm{~min}$ & $-1.4 \pm 10.3$ & $-0.4 \pm 6.0$ & $-0.2 \pm 1.2$ & 0.81 \\
& & & & \\
Acute (initial dose) & & & \\
$10 \mathrm{~s}$ & $38.3 \pm 77.6$ & $3.0 \pm 6.1$ & $-0.5 \pm 3.7$ & 0.005 \\
$3 \mathrm{~min}$ & $1.1 \pm 8.5$ & $1.0 \pm 2.5$ & $-0.8 \pm 2.9$ & 0.38 \\
$10 \mathrm{~min}$ & $0.3 \pm 5.2$ & $0.1 \pm 1.8$ & $-0.7 \pm 3.6$ & 0.65 \\
\hline
\end{tabular}


stinging $(P<0.001)$ and redness $(P=0.04)$ with dorzolamide having the higher value among the groups. Additionally, a difference in blurred vision existed between groups with both dorzolamide and brinzolamide having an increased incidence over placebo

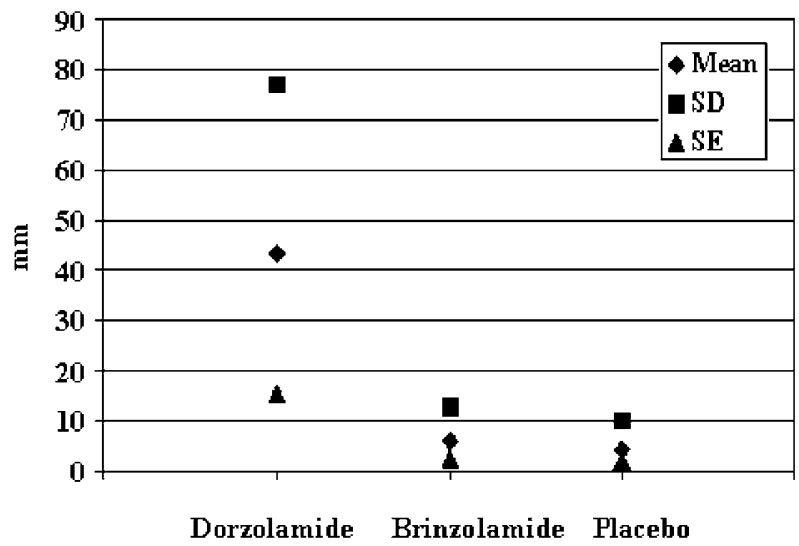

Figure 1 A modified box plot diagram showing the difference in pain scores between dorzolamide, brinzolamide, and placebo at $10 \mathrm{~s}$ after instillation for acute dosing.

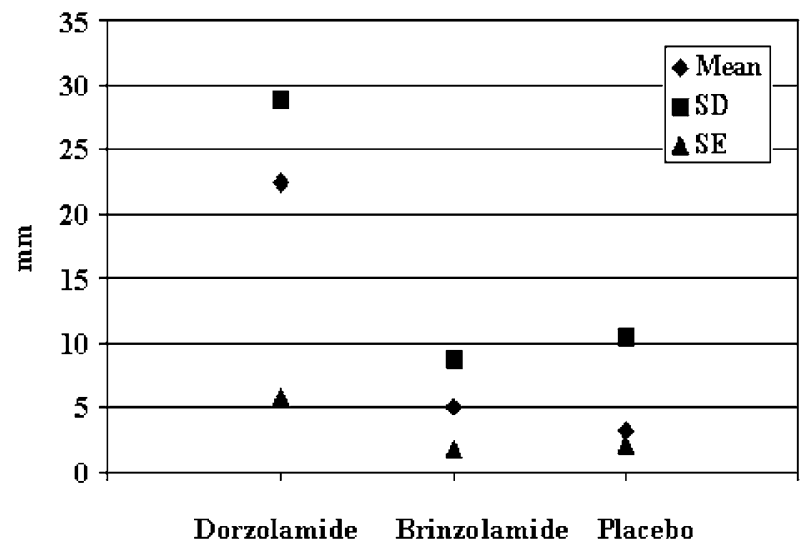

Figure 2 A modified box plot diagram showing the difference in pain scores between dorzolamide, brinzolamide, and placebo at $10 \mathrm{~s}$ after instillation for chronic dosing.
$(P=0.034)$. There was no significant difference between groups for foreign body sensation $(P=0.47)$, photophobia $(P=0.13)$, or dryness $(P=0.19)$.

On the systemic symptom query there was no significant difference between dorzolamide, brinzolamide, and placebo for any category including: fatigue, dizziness, despondency, depression, and taste perversion $(P>0.05)$.

The intraocular pressure at trough for brinzolamide was $19.7 \pm 2.2,19.3 \pm 1.8$ for dorzolamide and $21.1 \pm 2.6 \mathrm{mmHg}$ for placebo. This was a significant difference between groups $(P<0.05)$. The mean visual acuity values are shown in Table 4 . No significant difference between the groups $(P>0.1)$ was observed at

Table 3 Ocular symptoms query results $(n=25)$

\begin{tabular}{lrccc}
\hline & Absent & Mild & Moderate & Severe \\
\hline Dorzolamide & & & & \\
$\quad$ Foreign body sensation & 19 & 5 & 1 & 0 \\
Stinging/burning** & 9 & 9 & 5 & 2 \\
Redness** & 17 & 6 & 2 & 0 \\
Blurred vision* & 14 & 9 & 2 & 0 \\
Pain upon light exposure & 23 & 2 & 0 & 0 \\
Dryness & 21 & 3 & 1 & 0 \\
& & & & \\
Brinzolamide & & & & \\
Foreign body sensation & 19 & 4 & 2 & 0 \\
Stinging/burning** & 22 & 3 & 0 & 0 \\
Redness*** & 22 & 2 & 1 & 0 \\
Blurred vision* & 15 & 9 & 0 & 1 \\
Pain upon light exposure & 25 & 0 & 0 & 0 \\
Dryness & 19 & 6 & 0 & 0 \\
& & & & \\
Placebo & & & & \\
Foreign body sensation & 22 & 2 & 0 & 1 \\
Stinging/burning** & 25 & 0 & 0 & 0 \\
Redness*** & 25 & 0 & 0 & 0 \\
Blurred vision* & 22 & 3 & 0 & 0 \\
Pain upon light exposure & 25 & 0 & 0 & 0 \\
Dryness & 24 & 1 & 0 & 0 \\
\hline
\end{tabular}

${ }^{*} P=0.034,{ }^{* *} P<0.001,{ }^{* * *} P=0.04$

Table 4 Visual acuity (ETDRS) $(n=25)$

\begin{tabular}{lcccc}
\hline & Baseline & $1 \mathrm{~min}$ & $5 \mathrm{~min}$ & $12 \mathrm{~min}$ \\
\hline OD & & & & $-0.02 \pm 0.1$ \\
$\quad$ Dorzolamide & $-0.002 \pm 0.1$ & $-0.002 \pm 0.1$ & $-0.019 \pm 0.1$ & $-0.036 \pm 0.09$ \\
Brinzolamide & $0.004 \pm 0.1$ & $-0.002 \pm 0.09$ & $-0.014 \pm 0.09$ & $-0.03 \pm 0.1$ \\
Placebo & $-0.017 \pm 0.09$ & $-0.017 \pm 0.1$ & $-0.029 \pm 0.09$ & $-0.016 \pm 0.12$ \\
OS & & & & $-0.052 \pm 0.09$ \\
Dorzolamide & $-0.002 \pm 0.12$ & $0.009 \pm 0.13$ & $-0.008 \pm 0.12$ & $-0.034 \pm 0.1$ \\
Brinzolamide & $-0.009 \pm 0.08$ & $-0.033 \pm 0.08$ & $-0.034 \pm 0.09$ & $-0.038 \pm 0.09$ \\
Placebo & $-0.011 \pm 0.1$ & $-0.022 \pm 0.1$ & & \\
\hline
\end{tabular}

No significant differences were found between groups at each time point, $P>0.1$. 
baseline or any postinstillation time point in both right and left eyes.

Beyond symptoms reported on the quality of life surveys and the visual analogue scale, no adverse events were reported in this study.

\section{Discussion}

A clinical difference noted in the development of brinzolamide, compared to dorzolamide, was the lack of unsolicited complaints of stinging. ${ }^{15,16}$ In the regulatory trial by Silver and associates, ${ }^{15}$ when brinzolamide was used as monotherapy, there were statistically fewer nonsolicited complaints of ocular stinging upon instillation compared to dorzolamide monotherapy. Any stinging in this study was characterized as mild with both topical carbonic anhydrase inhibitors. Other complaints, occurring at higher than the 3\% level, were blurred vision with brinzolamide, conjunctivitis with dorzolamide, and taste abnormality with all products, though there was no statistical difference between groups.

A similar study, by Michaud et al, ${ }^{16}$ compared brinzolamide $v s$ dorzolamide added to timolol. This study also showed statistically less frequent reports of nonsolicited stinging and burning with brinzolamide compared to dorzolamide. Taste abnormality was the only other event that occurred with greater than a $1 \%$ incidence.

In a separate study by Stewart and associates, burning and stinging were further evaluated with a solicited symptom survey, which ranked the discomfort between 0 (no discomfort) and 4 (severe discomfort). Brinzolamide showed statistically less incidences of burning and stinging, averaging 0.2-0.4 compared to dorzolamide at 1.5-1.7. ${ }^{9}$ Further, Barnebey and Kwok ${ }^{17}$ reported the results of a multicentre switch study, which indicated that $69 \%$ of patients had improvement in their comfort rating after switching to brinzolamide.

Despite the above, there is little information that compares the ocular discomfort between these two medicines using numeric measuring scales or solicited symptom queries.

This current study evaluated the short-term ocular tolerability of dorzolamide $2 \%$ vs brinzolamide $1 \%$ both given three times daily using a numeric visual analogue pain scale and solicited subject complaints.

This study found that within the first $10 \mathrm{~min}$ after dosing that there was a significant difference in ocular pain between placebo, brinzolamide, and dorzolamide at 10 -s postinstillation on the visual analogue pain scale. At this time point dorzolamide had the highest levels. However, no difference between groups was observed preinstillation, after 3 days of dosing, nor at 3 or $10 \mathrm{~min}$ postdosing. Additionally, the change from baseline was significantly different between groups only at 10 -s postinstillation.

The highest mean level of pain recorded was 22 out of 100 (' 0 ' represented no pain and ' 100 ' represented the worst imaginable pain) $10 \mathrm{~s}$ after instillation with dorzolamide. All other recordings were close to the levels of the placebo and brinzolamide, less than a reading of 10. Previous investigators have tried to associate the numeric pain scale to a subjective intensity of pain. Moderate pain was noted in these studies to be $30-75 \mathrm{~mm}$ or above. ${ }^{18-22}$ The pain value found with dorzolamide in this study was less than that found with timolol maleate that measured $27 \mathrm{~mm}+$, using the same scale and time points, in a study by Scoville et $a l^{10}$ a decade ago.

On first instillation of study medicine dorzolamide also caused statistically more ocular pain than brinzolamide or placebo. This occurred only at the $10 \mathrm{~s}$ time point postdosing, similar to after 3 days dosing. However, the average pain was higher on the initial drop (43.3) than after 3 days of dosing (22.5). This indicates that the ocular stinging subjects experience on initial instillation may then abate after a few days of chronic dosing.

The ocular symptom query evaluated the subject's global tolerability after 3 days of chronic dosing. Burning/stinging and redness were significantly greater with dorzolamide than brinzolamide or placebo. In most cases the symptoms were classified as 'mild' and did not lead to discontinuation of the medicine in any case. Other symptoms (such as foreign body sensation, photophobia, and dryness) were not significantly different between groups.

Blurred vision was significantly different between groups but was assessed by subjects to be similar between dorzolamide and brinzolamide. Despite the symptom of blurred vision, however, no mean change in vision from placebo was measured postinstillation. Systemic symptoms, including fatigue, dizziness, despondency, depression, and taste perversion were statistically similar between groups.

Intraocular pressure was found to be similar between the three study groups. However, this study was not the best measure in change from baseline because of the short washout time between the screening and baseline visits. Previous therapy with a medication requiring long washout time (eg, timolol or latanoprost) could have influenced the intraocular pressure during this trial. Nonetheless the pressures of both active medicines in this trial were similar, as noted in past reports, and were reduced from placebo. ${ }^{15,16}$

This study suggests that subjects treated with dorzolamide suffer more ocular pain lasting for at least $10 \mathrm{~s}$ upon instillation compared to brinzolamide and 
placebo. The pain is characterized as burning and stinging, which is generally mild and short in duration. Further, ocular pain on initial dosing with dorzolamide may abate somewhat after a few days of dosing.

This study did not evaluate the cause of the burning with dorzolamide. This potentially could result from the compound itself or the lower $\mathrm{pH}$ compared to the other two formulations evaluated in this study. Also, this study did not evaluate differences in long-term tolerability between dorzolamide and brinzolamide or with twice daily dosing. Further study may clarify comfort differences between dorzolamide and brinzolamide.

\section{References}

1 Lippa EA, Carlson LE, Ehinger B, Eriksson LO, Finnstrom $\mathrm{K}$, Holmin $\mathrm{C}$ et al. Dose response and duration of action of dorzolamide, a topical carbonic anhydrase inhibitor. Arch Ophthalmol 1992; 110: 495.

2 Lippa EA, Schuman JS, Higginbotham EJ, Kass MA, Weinrib RN, Skuta GL et al. MK-507 versus sezolamide: a comparative efficacy of two topically active carbonic anhydrase inhibitors. Ophthalmology 1991; 98(3): 308.

3 Wilkerson M, Cyrlin M, Lippa EA, Esposito D, Deasy D, Panebianco D et al. Four-week safety and efficacy study of dorzolamide, a novel, active topical carbonic anhydrase inhibitor. Arch Ophthalmol 1993; 111: 1343.

4 Kitazawa Y, Azuma I, Iwata K, Tsukahara S, Shioes Y, Araie $\mathrm{M}$ et al. Dorzolamide, a topical carbonic anhydrase inhibitor: a two-week dose response study in patients with glaucoma or ocular hypertension. J Glaucoma 1994; 3: 275-279.

5 Strahlman E, Tipping R, Vogel R, the International Dorzolamide Study Group. A double masked, randomized 1-year study comparing dorzolamide (Trusopt), timolol, and betaxolol. Arch Ophthalmol 1995; 113: 1009-1016.

6 Laibovitz R, Strahlman ER, Barber BL, Strohmaier KM. Comparison of quality of life and patient preference of dorzolamide and pilocarpine as adjunctive therapy to timolol in the treatment of glaucoma. J Glaucoma 1995; 4: 306-313.

7 Shin DH, the Brinzolamide Adjunctive Therapy Study Group. A triple-masked, placebo-controlled, adjunctive therapy study of the efficacy and safety of TID-dosed brinzolamide $1.0 \%$ compared to TID-dosed placebo when used adjunctively to timolol $0.5 \%$. Invest Ophthalmol Vis Sci (Suppl) 1997; 38: 2605.

8 Camras CB, the Brinzolamide Primary Therapy Study Group. A triple-masked, primary therapy study of the efficacy and safety of BID and TID-dosed brinzolamide 1\% compared to TID-dosed dorzolamide $2 \%$ and BID-dosed timolol 0.5\%. Invest Ophthalmol Vis Sci (Suppl) 1997; 38: 2605.

9 Stewart R, the Brinzolamide Comfort Study Group. The ocular comfort of the TID-dosed brinzolamide $1.0 \%$ compared to TID-dosed dorzolamide $2.0 \%$ in patients with primary open-angle glaucoma or ocular hypertension. Invest Ophthalmol Vis Sci (Suppl) 1997; 38: 2603.

10 Scoville B, Krieglstein GK, Then E, Yokoyama S, Yokoyama T. Measuring drug-induced eye irritation: a simple new clinical assay. J Clin Pharmacol 1985; 25: 210-218.

11 Price DD, McGrath PA, Rafii A, Buckingham B. The validation of visual analogue scales as ratio scale measures for chronic and experimental pain. Pain 1983; 17: 45-56.

12 Book SA. Essentials of Statistics. McGraw Hill Book Company: New York, 1978, pp 117-122, 205-215.

13 Rosner B. Fundamentals of Biostatistics. Duxbury Press: Boston, 1982, pp 336-341.

14 Siegel S. Nonparametric Statistics for the Behavioral Sciences. McGraw Hill Book Company: New York, 1956, pp 63-67.

15 Silver LH, the Brinzolamide Primary Therapy Study Group. Clinical efficacy and safety of brinzolamide (Azopt ${ }^{\mathrm{TM}}$ ), a new topical carbonic anhydrase inhibitor for primary openangle glaucoma and ocular hypertension. Am J Ophthalmol 1988; 126: 400-408.

16 Michaud JE, Friren B, the International Brinzolamide Adjunctive Study Group. Comparison of topical brinzolamide $1 \%$ and dorzolamide $2 \%$ eye drops given twice daily in addition to timolol $0.5 \%$ in patients with primary open-angle glaucoma or ocular hypertension. Am J Ophthalmol 2001; 132: 235-243.

17 Barnebey H, Kwok SY. Patients' acceptance of a switch from dorzolamide to brinzolamide for the treatment of glaucoma in a clinical practice setting. Clin Ther 2000; 22: 1204-1212.

18 Sriwatanakul K, Kelvie W, Lasagna L, Calimlim JF, Weis OF, Mehta G. Studies with different types of visual analog scales for measurement of pain. Clin Pharmacol Ther 1983; 34(2): 234-239.

19 Collins SL, Moore RA, McQuay HJ. The visual analogue pain intensity scale: what is moderate pain in millimeters? Pain 1997; 72(1-2): 95-97.

20 Curtis P, Gartman LA, Green DB. Utilization of ketorolac tromethanine for control of severe odontogenic pain. J Endod 1994; 20(9): 457-459.

21 Seymour RA, Kelly PJ, Hawesford JE. The efficacy of ketoprofen and paracetamol (acetaminophen) in postoperative pain after third molar surgery. $\mathrm{Br} \mathrm{J}$ Clin Pharmacol 1996; 41: 581-585.

22 Stubhaug A, Grimstad J, Breivik H. Lack of analgestic effect of 50 and $100 \mathrm{mg}$ oral tramadol after orthopedic surgery: a randomized, double-blind, placebo and standard active drug comparison. Pain 1995; 62(1): 111-118. 